Research Article

\title{
Miscibility gap alloys with a ceramic matrix for thermal energy storage
}

\author{
Samuel Reed ${ }^{1}$ (D) $\cdot$ Erich Kisi $^{1}$
}

Received: 23 June 2020 / Accepted: 21 November 2020 / Published online: 4 December 2020

(c) Springer Nature Switzerland AG 2020

\begin{abstract}
New miscibility gap alloys with a ceramic matrix have been explored in the $\mathrm{ZrO}_{2}-\mathrm{Al}, \mathrm{AIN}-\mathrm{Al}, \mathrm{AIN}-(\mathrm{Al}-\mathrm{Si}), \mathrm{Al}_{2} \mathrm{O}_{3}-\mathrm{Al}$ and $\mathrm{MgO}-\mathrm{Al}$ systems with a view to creating oxidation-resistant macroscopically solid, phase change-enhanced, thermal energy storage materials. Materials were manufactured by mixing the components, pressing and firing at $700{ }^{\circ} \mathrm{C}$ under argon. Oxidation tests at $700{ }^{\circ} \mathrm{C}$ in air for up to $72 \mathrm{~h}$ showed a mix of responses. Despite favourable thermodynamic properties of some published Ellingham diagrams, samples in the $\mathrm{ZrO}_{2}-\mathrm{Al}$ system underwent a strong exchange reaction, which did not result in a useful material. In the AIN-Al, AIN-(AI-Si) systems, viable MGA-type materials with encapsulated Al particles providing latent heat storage within a solid matrix were formed. There was no degradation of the materials after $24 \mathrm{~h}$ of oxidation testing; however, severe oxidation of the $\mathrm{Al}$ or Al-Si was observed after $72 \mathrm{~h}$. The $\mathrm{Al}_{2} \mathrm{O}_{3}-\mathrm{Al}$ and $\mathrm{MgO}-\mathrm{Al}$ systems not only formed a viable ceramic matrix material after firing, but also showed no signs of Al oxidation after $72 \mathrm{~h}$ in air at $700^{\circ} \mathrm{C}$. These results open up a promising new series of thermal energy storage materials, some of which appear to have very good oxidation resistance under the test conditions.
\end{abstract}

Keywords Thermal energy storage $\cdot$ Miscibility gap alloys $\cdot$ Phase change materials $\cdot$ High-temperature storage $\cdot$ Oxygen resistant $\cdot$ High thermal conductivity $\cdot$ Differential thermal analysis $\cdot X$-ray diffraction

\section{Introduction}

In an environment of extreme downward pressure on fossil fuel power production and abundant and relatively cheap renewable energy sources from the sun and wind, economically viable energy storage is desperately needed due to the intermittent nature of the latter two. Within the wide spectrum of energy storage technologies available, thermal energy storage has a number of potential advantages, which have been identified and discussed widely in the literature. Approximately half of the energy used by humanity is used as heat. Sources of heat such as concentrated sunlight or industrial waste heat are abundant, and conversion from electrical to thermal energy is rapid and very efficient. At large scale, the losses from thermal energy storage can be as low as a few $\%$ per day [1-3].
The storage of thermal energy may be accomplished in several ways. Primarily, a heated body of solid or liquid stores sensible heat at a rate that is approximately linear with temperature. Alternatively, a material that undergoes a phase change within the temperature range of interest stores primarily latent heat if operated over a narrow temperature range around the melting temperature, hence the term phase change material (PCM). Materials and systems for sensible heat storage and latent heat storage have been extensively studied and reviewed [1-9]. State of the art is molten salt storage, which uses a variety of nitrate salts pumped through heat exchangers between hot and cold storage tanks $[4,10,11]$.

Miscibility gap alloys (MGA) form a class of high thermal conductivity solids enhanced by an internal PCM dispersed as fine particles and fully encapsulated within $[12,13]$. Although the PCM melts and stores latent heat,

$\triangle$ Samuel Reed, samuel.reed@uon.edu.au| 'School of Engineering, The University of Newcastle, Callaghan, Newcastle, NSW 2308, Australia.

SN Applied Sciences (2020) 2:2148 | https://doi.org/10.1007/s42452-020-03942-1 
the materials remain macroscopically solid and immobile. This is a key attribute of MGA modules, as the MGA systems absorb energy like a PCM, but they do not require additional costly encapsulation techniques. MGA modules offer a number of advantages over sensible heat storage and molten salt storage in particular including greater system simplicity, no parasitic energy use to keep the system molten, higher temperatures accessible, a concentration of energy around the phase change temperature and the use of simple and environmentally benign materials [14]. Initial MGA were made from two metals (Al-Sn, Fe-Cu, Fe-Mg, etc.); however, recently a number of graphite matrix MGA have been manufactured and tested in relatively arduous conditions [15]. These systems include C-Zn [15], C-Al and C-(Al-Si) [16, 17], C-Mg and C-Cu [13].

The performance of these thermal storage materials under the test conditions has shown considerable promise for a number of practical applications including building and district heating as well as the purpose for which they were originally developed, concentrated solar thermal power generation (CSP). In practical terms, these MGA have survived more than 200 charge-discharge cycles [17], survived direct solar irradiation [15] and survived a combination of direct irradiation with energy extraction using an embedded water pipe [18]. One minor limitation of the materials is that, above $400^{\circ} \mathrm{C}$, they require protection from oxidation through the use of a protective atmosphere (typically $\mathrm{N}, \mathrm{Ar}$ or $\mathrm{CO}_{2}$ ).

As the matrix component of an MGA fully encapsulates the metallic PCM particles inside, the oxidation resistance is determined by the matrix component. Ceramic materials are either already oxides or generally have superior oxidation resistance to metals and graphite. Consequently, it is of considerable interest to ascertain whether viable MGA materials can be fabricated using commonly available ceramics, with the addition of low-cost metal particles such as Al.

The primary requirement for successful manufacture of an MGA is that the components are neither soluble nor chemically reactive with each other under the manufacturing or operational conditions. Consultation of the appropriate Ellingham diagram shows that for Al particles as the dispersed PCM, some metal oxides such as $\mathrm{Fe}_{2} \mathrm{O}_{3}$ are clearly unstable, whereas some, such as $\mathrm{MgO}$, are thermodynamically stable. Some oxides such as $\mathrm{ZrO}_{2}$ may be marginally unstable against an oxygen exchange reaction below $730^{\circ} \mathrm{C}$ although published Ellingham diagrams differ somewhat in this [19-21]. In addition, ceramics based on $\mathrm{Al}$ metal such as $\mathrm{Al}_{2} \mathrm{O}_{3}$ or AIN have no net reaction available and are expected to be stable. In addition to thermodynamic considerations, a system may be trapped in a thermodynamically unfavourable state by kinetic limitations. This is the case for the C-AI MGA system in which it has been shown that the formation of aluminium carbide can be avoided $[16,17]$.

The second requirement is that the two components have negligible miscibility at the operational temperatures. Metals and ceramics are generally immiscible, and therefore, this criterion is easily met. Thirdly, it is essential that the PCM component is well dispersed within the matrix component in a non-percolating arrangement so that it cannot flow out of the MGA when it is above the PCM melting temperature.

Based upon these criteria, it was decided that an initial exploration into the manufacture of MGA materials using $\mathrm{ZrO}_{2}, \mathrm{AIN}, \mathrm{Al}_{2} \mathrm{O}_{3}$ and $\mathrm{MgO}$ as the ceramic matrix would be undertaken. In this paper are reported the results of that manufacture and subsequent oxidation testing of trial ceramic-matrix MGA using four ceramic matrix components and particulate $\mathrm{Al}$ as the dispersed metallic PCM component as well as one system using dispersed $\mathrm{Al}-12.7 \mathrm{wt} \% \mathrm{Si}$.

\section{Experimental methods}

The MGA systems chosen for study were $\mathrm{ZrO}_{2}-\mathrm{Al}$, AIN-Al, AIN-(Al-Si), $\mathrm{Al}_{2} \mathrm{O}_{3}-\mathrm{Al}$ and MgO-Al. Ceramic powders selected were $\mathrm{ZrO}_{2}$ (5 $\mu \mathrm{m}$, Sigma-Aldrich), AIN (10 $\mu \mathrm{m}$, Chengdu Huarui Industrial Co., Ltd.), $\mathrm{Al}_{2} \mathrm{O}_{3}(<45 \mu \mathrm{m}$, Univar) and $\mathrm{MgO}(<45 \mu \mathrm{m}$, Thermo Fisher Scientific Inc.) being examined. Al ( $<75 \mu \mathrm{m}$, Alloys International) and $\mathrm{Al}-\mathrm{Si}(<90 \mu \mathrm{m}$, Oerlikon Metco) were used as active phase materials. Powders of the respective components were first mixed at $50 \%$ by volume in a SPEX high-energy ball mill for a duration of $15 \mathrm{~min}$. A charge ratio of 10:1 (mass of steel balls to powder mass) was used followed by uniaxial pressing in a 15-mm-diameter die under a 5-tonne load. The pressing process provided "green pellets" which were then ready to be fired at a nominated temperature. Samples were fired in batches of three identical pellets using an inert atmosphere (Ar flow) at a temperature of $700^{\circ} \mathrm{C}$ in a horizontal tube furnace. This temperature was chosen for compatibility with previous C-Al experiments [16] and to avoid the viscosity of the melted Al particles becoming too low which it was felt may cause some leakage in the early stages of firing. This treatment is insufficient to cause full sintering of the pure ceramics which generally requires heating in the range $1200-1800^{\circ} \mathrm{C}$.

Oxidation tests were carried out in an oxygen-rich environment within a laboratory-scale muffle furnace. Heating was undertaken at $5^{\circ} \mathrm{C} / \mathrm{min}$ until the hold temperature of $700{ }^{\circ} \mathrm{C}$ was reached. MGA samples were extracted from the furnace at intervals which included: $2 \mathrm{~h}, 8 \mathrm{~h}, 24 \mathrm{~h}$, $48 \mathrm{~h}$ and $72 \mathrm{~h}$. At these intervals, sample masses were recorded along with $\mathrm{X}$-ray diffraction patterns to observe 
any oxidation of the AI PCM particles. No weight changes were noted throughout the experimental stages.

$\mathrm{X}$-ray diffraction (XRD) was used to determine the phase composition of the systems with a PANalytical X-Pert Pro diffractometer using $\mathrm{Cu} \mathrm{K}_{\alpha}$ radiation within a scanning angle of $2 \theta=15-75^{\circ}$. XRD phase analysis was conducted by utilising the International Centre of Diffraction Database (ICDD).

Differential thermal analysis (DTA) was conducted in a vertical tube furnace, utilising a purpose built apparatus that enables the recording of data from MGA samples that are $15 \mathrm{~mm}$ in diameter [17]. To conduct the tests, samples were heated to $750^{\circ} \mathrm{C}$ in an argon (Ar)-rich environment and at a heating rate of $5{ }^{\circ} \mathrm{C} / \mathrm{min}$. A pure copper $(\mathrm{Cu})$ sample was used as a reference as it does not undergo any transitions in this temperature range. DTA was used to investigate both as-fired MGA samples as well as those that had undergone oxidation testing. Samples for microscopy were set in resin and polished to a sub one-micron finish for optical microscopy.

\section{Results}

Upon removal from the furnace, the $\mathrm{ZrO}_{2}$ ceramic matrix MGA had suffered severe deformation along with cracking and swelling after the firing treatment making this system unsuitable for further testing. XRD analysis (Fig. 1) indicated that an exchange reaction had occurred between the $\mathrm{ZrO}_{2}$ and $\mathrm{Al}$ forming $\mathrm{ZrAl}$, which is a known to occur with a 50:50 atomic per cent between $\mathrm{Al}$ and $\mathrm{Zr}$ and $\mathrm{Al}_{2} \mathrm{O}_{3}$. It was thought prior to this experiment that $\mathrm{ZrO}_{2}$ would be more stable than the kinetics required to form a transformation between $\mathrm{Zr}$ and Al. Major peaks of ZrAl are represented by markers in Fig. 1, with minor reflections also being present at $2 \theta=20.26^{\circ}, 21.89^{\circ}, 50.74^{\circ}$ and $56^{\circ}$. Along with major reflections, minor reflections of $\mathrm{Al}_{2} \mathrm{O}_{3}$ are witnessed at $2 \theta=35^{\circ}, 52.5^{\circ}$ and $66.5^{\circ}$. The combination of these factors was sufficient to rule out further $\mathrm{ZrO}_{2}$ testing at this stage due to the matrix materials compatibility with AI PCM particles at the nominated volumetric ratio.

The other systems fared better and samples were examined in more detail using differential thermal analysis, optical microscopy and XRD phase analysis as appropriate.

\subsection{Thermal analysis}

The behaviour of the AIN-Al and AIN-(Al 12.7\%Si) systems was similar in all regards, and so, statements concerning the former may be inferred to apply to the latter also. The fired AIN-Al samples were mid-grey in colour with a slightly powdery exterior upon removal from the furnace after firing. There was minimal evidence for leakage of $\mathrm{Al}$ out of the samples. Visual inspection revealed no cracking or other macroscopic defects. Typical differential thermal analysis (DTA) traces for $\mathrm{AlN}-\mathrm{Al}_{1} \mathrm{Al}_{2} \mathrm{O}_{3}-\mathrm{Al}$ and $\mathrm{MgO}-\mathrm{Al}$ samples recorded during heating to $755^{\circ} \mathrm{C}$ are shown in Fig. 2 . These data were recorded on an in-house constructed DTA instrument specifically designed for large samples. As discussed in an earlier publication [17], conventional DSC and DTA instruments are not suitable for studying MGA samples as the material is inhomogeneous over length scales much larger than the conventional sample size (20-50 mg).

In each part of Fig. 2, the solid curves are for as-fired samples and the dashed curves are for samples given an oxidising treatment in air for $72 \mathrm{~h}$ at $700^{\circ} \mathrm{C}$. In each curve, the melting transition starts at the melting temperature of $\mathrm{Al}\left(660^{\circ} \mathrm{C}\right)$ and displays a large endotherm indicating suitability for thermal energy storage. However, the AIN-Al sample after oxidation for $72 \mathrm{~h}$ has a greatly reduced thermal response suggesting poor long-term oxidation resistance at this temperature (see also Sect. 3.2).

Upon removal of the $\mathrm{Al}_{2} \mathrm{O}_{3}$ and $\mathrm{MgO}$ matrix MGA pellets from the firing furnace, it was found that little visible change had occurred. In contrast to the AIN-Al systems,
Fig. 1 XRD pattern from $\mathrm{ZrO}_{2}-$ Al MGA after firing at $700{ }^{\circ} \mathrm{C}$

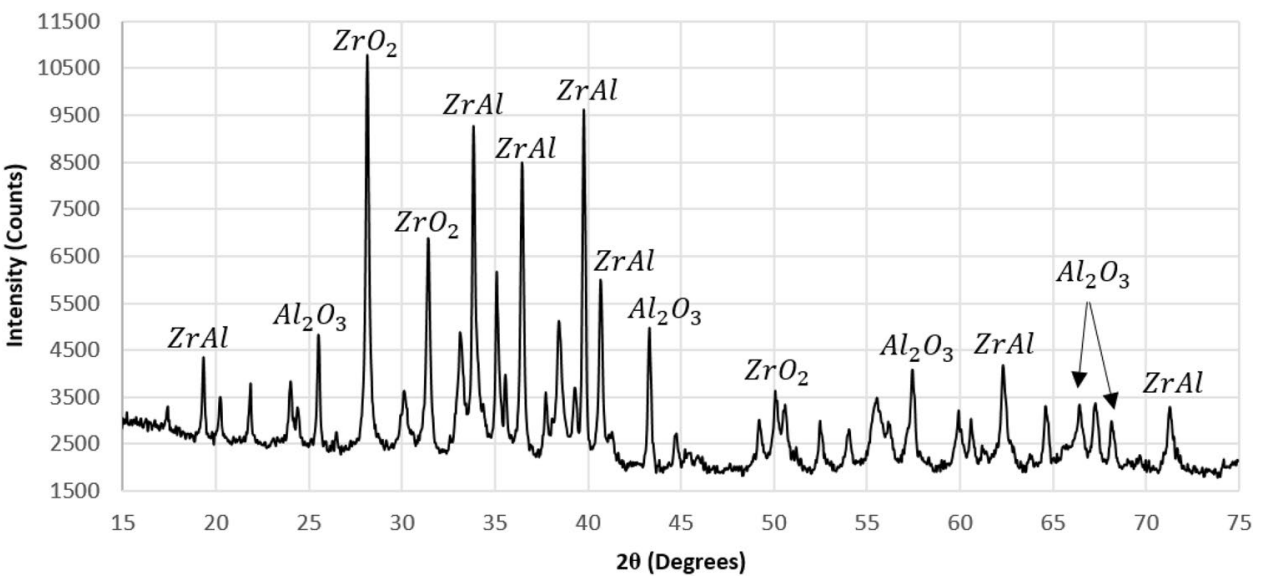

SN Applied Sciences A SPRINGER NATURE journa 

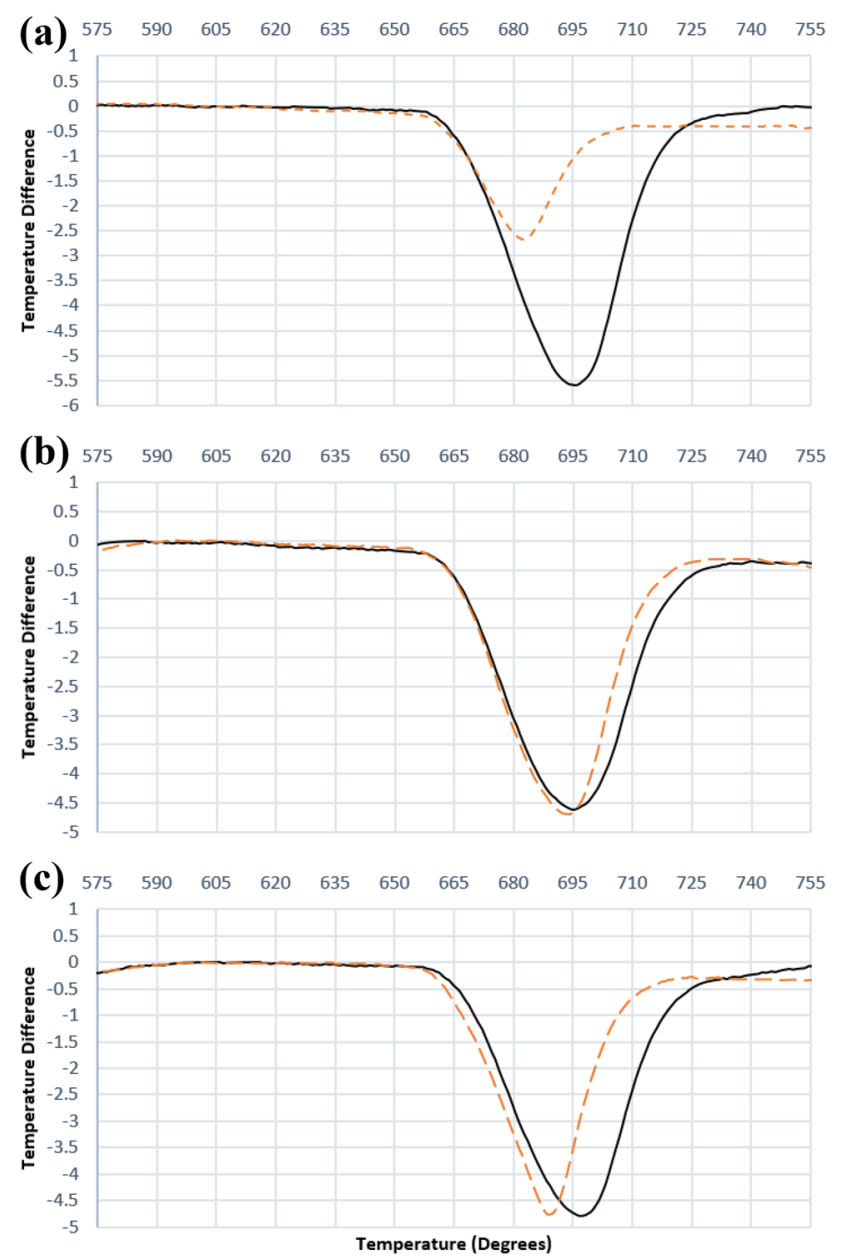

Fig. 2 DTA responses from sintered (solid) and oxidised (dashed) a AIN-Al b MgO-Al and c $\mathrm{Al}_{2} \mathrm{O}_{3}-\mathrm{Al}$ MGA samples during heating to $755^{\circ} \mathrm{C}$

the endotherms from the $\mathrm{Al}_{2} \mathrm{O}_{3}-\mathrm{Al}$ and $\mathrm{MgO}-\mathrm{Al}$ samples before and after the oxidation treatment are very similar within the precision of this large-sample DTA apparatus, suggesting that they have good oxidation resistance under the conditions employed in this work.

\subsection{Phase composition}

\subsubsection{AIN-Al and AIN-(AI-Si)}

A typical X-ray diffraction pattern from the surface of an as-fired AIN-Al sample after lightly abrading with 1000grit $\mathrm{SiC}$ paper is shown in Fig. 3a. Only peaks from the two primary constituents, AIN and Al, are visible. The pattern in Fig. $3 \mathrm{~b}$ is typical of samples after $24 \mathrm{~h}$ in air at $700{ }^{\circ} \mathrm{C}$ and shows diffraction peaks only from the two constituent materials AIN and Al. The AIN-(AI-Si) MGA showed the same results with only AIN, Al and Si diffraction peaks. Therefore, both variants exhibit excellent short-term oxidation resistance $\left(<24 \mathrm{~h}\right.$ at $\left.700^{\circ} \mathrm{C}\right)$. However, as shown in Fig. 4, longer-term exposure to air at $700^{\circ} \mathrm{C}$ caused considerable oxidation of the $\mathrm{Al}$ and $\mathrm{AIN}$, both changing to $\mathrm{Al}_{2} \mathrm{O}_{3}$, which suggests that AIN is not suitable for longterm storage in an air environment. This supports the DTA data presented in $\$ 3.1$.

\section{3 $\mathrm{Al}_{2} \mathrm{O}_{3}-\mathrm{Al}$ and $\mathrm{MgO}-\mathrm{Al}$}

Optical microscopy was used to investigate the microstructure of these two apparently oxidation-resistant systems. Figure 5 shows a typical image of the $\mathrm{MgO}-\mathrm{Al}$ microstructure.

This is a typical MGA microstructure with a high volume fraction of discrete PCM (Al) particles, well separated by the matrix phase $(\mathrm{MgO})$ as seen in previous work [12, $13,16]$. No evidence for microstructural degradation was found.

The phase assembly was later investigated using XRD analysis. XRD results for $\mathrm{MgO}-\mathrm{Al}$ and $\mathrm{Al}_{2} \mathrm{O}_{3}-\mathrm{Al}$ samples in the as-fired condition and after they were held at $700^{\circ} \mathrm{C}$ in air for $72 \mathrm{~h}$ are shown in Figs. 6 and 7. From a visual comparison of Fig. 6a with Figs. $6 \mathrm{~b}$ (MgO-Al) and 7a) with 7b) $\left(\mathrm{Al}_{2} \mathrm{O}_{3}-\mathrm{Al}\right)$, it is clear that there is no change to the samples by either oxidation of $\mathrm{Al}$ to $\mathrm{Al}_{2} \mathrm{O}_{3}$ or a reaction between $\mathrm{Al}$ and $\mathrm{MgO}$ to form intermediate compounds. Some changes to individual XRD peaks do occur; however, these are due to sampling statistics. A few large Al crystals can artificially increase the size of one peak due to favourable alignment. Whole pattern analysis using the Rietveld method was used to ensure that the phase proportions were the same before and after oxidation within the measurement error.

\subsection{Predicted properties}

Theoretical values for the room-temperature physical properties of the new materials are summarised in Table 1. Actual values at full-scale production may differ from these values due to trapped porosity or minor deviations of the microstructure from the ideal. As previously discussed [22], scalar properties such as density and specific heat are calculated using the appropriately weighted average of the constituent material properties. Transport properties are more complex. Thermal conductivity was calculated using the equation given by Maxwell (1893) for the electrical resistivity of a composite material comprising non-interacting spherical inclusions. This has been shown to give reasonable estimates for this kind of microstructure [22].

The expected temperature variation of the specific heat for the three materials is shown in Fig. 8 using data from $[23,24]$.

Figure 9 illustrates the theoretical stored energy density as a function of temperature from an assumed lower storage 
Fig. 3 a) AIN-(Al-Si) as-fired and b) AIN-(Al-Si) after 24-h oxidation at $700^{\circ} \mathrm{C}$
Fig. $4 \mathrm{Cu} k_{\alpha} \mathrm{X}$-ray diffraction pattern from the AIN-AI MGA oxidised for $72 \mathrm{~h}$ in air at $700{ }^{\circ} \mathrm{C}$ (a)
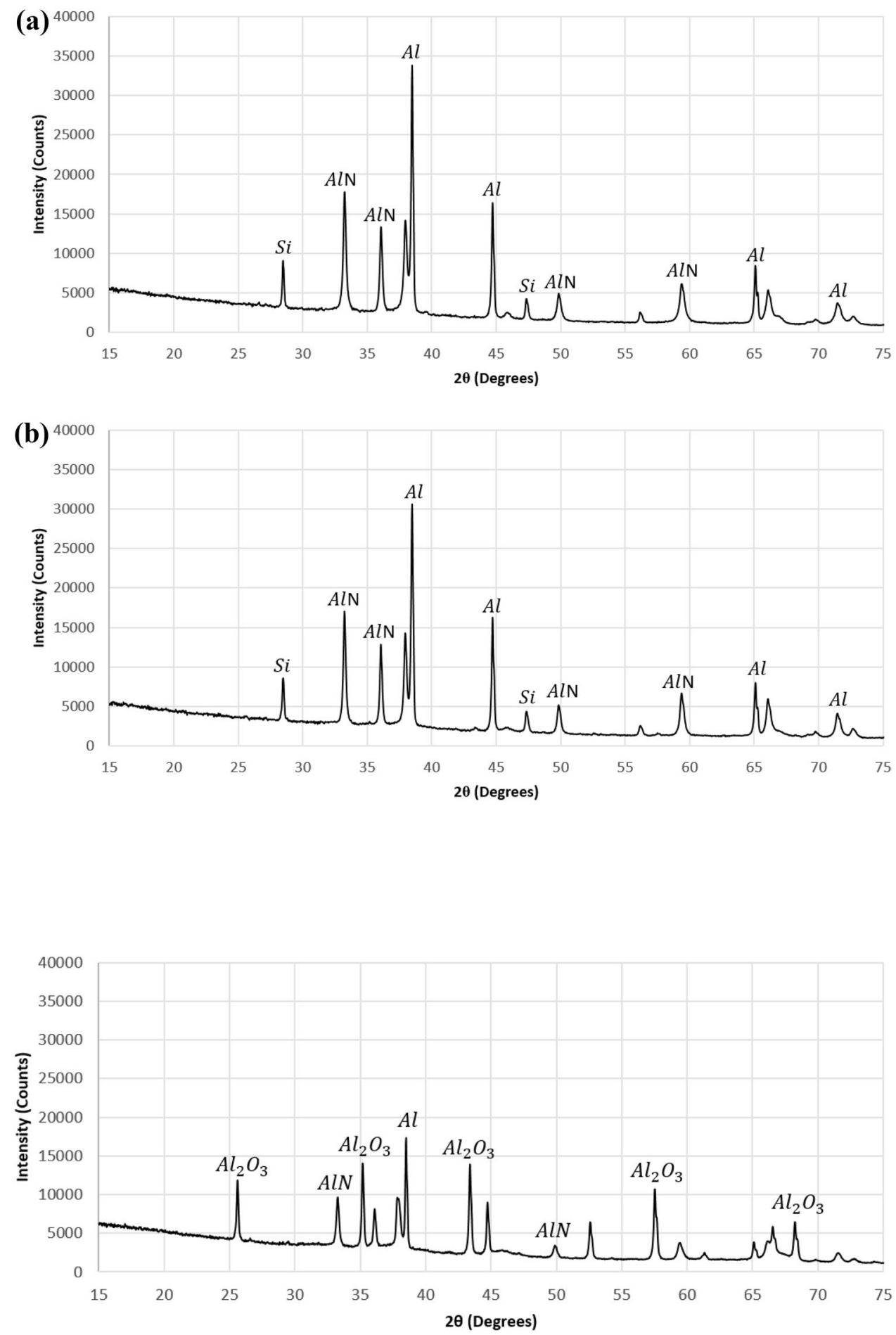

temperature of $610^{\circ} \mathrm{C}$ to an assumed upper temperature of $710^{\circ} \mathrm{C}$. These limits were chosen to match those used to describe the energy density of MGA materials elsewhere [13, $16,17,25]$ and do not indicate any restrictions on the temperature at which the materials can be used.

\section{Discussion}

Oxidation of TES materials such as nitrate salts, graphite and halide salts presents technical challenges to their 


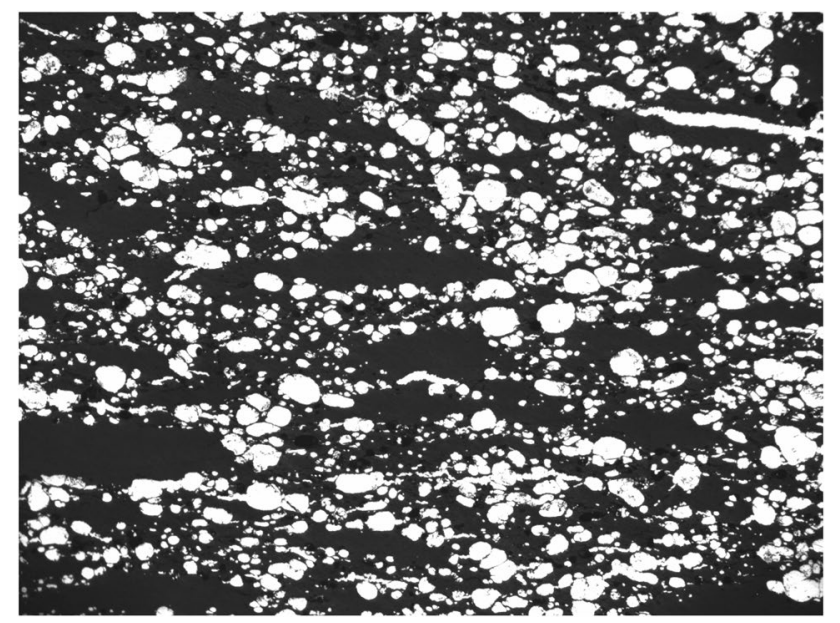

Fig. 5 Optical micrograph of an MgO-Al sample after oxidation for $72 \mathrm{~h}$ in air at $700{ }^{\circ} \mathrm{C}$. Al particles are bright, and the $\mathrm{MgO}$ matrix is dark

use which is then restricted to controlled atmosphere environments. The same is true to varying degrees for the higher-temperature examples of MGA systems developed to date. In graphite and graphite-based MGA systems, reaction between atmospheric oxygen and carbon begins at temperatures between 400 and $500^{\circ} \mathrm{C}$ depending on the density of the material, its surface structure and the gas flow conditions prevailing. This means that, as for other candidates, for carbon-based MGA to be successful TES media in CSP plants, a protective environment is required. To nullify this complication and reduce the overall cost of the TES, oxygen-resistant systems were investigated. As a general category of materials, ceramics possess excellent oxygen-resistant properties and were therefore chosen for investigation.

Four ceramic systems were investigated in this work, two of which showed promise as oxygen-resistant MGA. The $\mathrm{ZrO}_{2}$ matrix MGA was deemed unsuitable due to the oxygen exchange reaction, which resulted in reduction of $\mathrm{ZrO}_{2}$, transformation of the liberated $\mathrm{Zr}$ to $\mathrm{ZrAl}$ and the oxidation of $\mathrm{Al}$ to $\mathrm{Al}_{2} \mathrm{O}_{3}$ upon heating. The AIN matrix MGA is predicted to have excellent thermal conductivity; however, despite no signs of oxidation after $24 \mathrm{~h}$ at $700^{\circ} \mathrm{C}$, its long-term use in an air environment was not successful and considerable oxidation was observed after $72 \mathrm{~h}$ at $700{ }^{\circ} \mathrm{C}$. This is most probably due to a small fraction of open pores enabling oxygen to penetrate the MGA. This resulted in $\mathrm{Al}$ being oxidised to $\mathrm{Al}_{2} \mathrm{O}_{3}$. The AIN-AI MGA
Fig. $6 \mathrm{Cu} k_{\alpha} \mathrm{XRD}$ pattern from $\mathrm{MgO}-\mathrm{Al} \mathrm{MGA} \mathbf{a}$ as-fired and $\mathbf{b}$ oxidised for $72 \mathrm{~h}$ in air at $700^{\circ} \mathrm{C}$
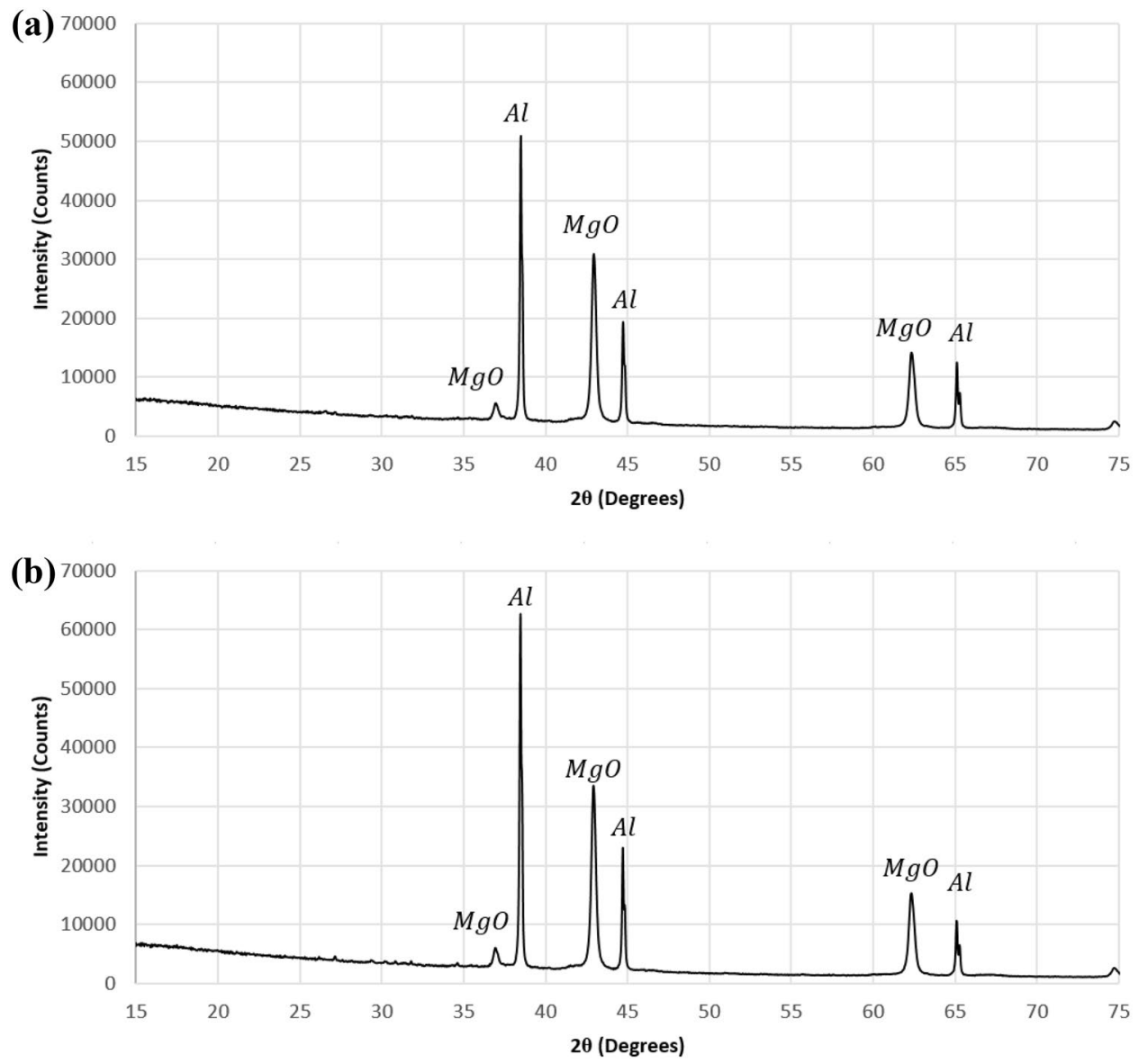
Fig. $7 \mathrm{Cu} k_{\alpha}$ XRD pattern from $\mathrm{Al}_{2} \mathrm{O}_{3}-\mathrm{Al} M G A$ a as-fired and $\mathbf{b}$ oxidised for $72 \mathrm{~h}$ in air at $700^{\circ} \mathrm{C}$

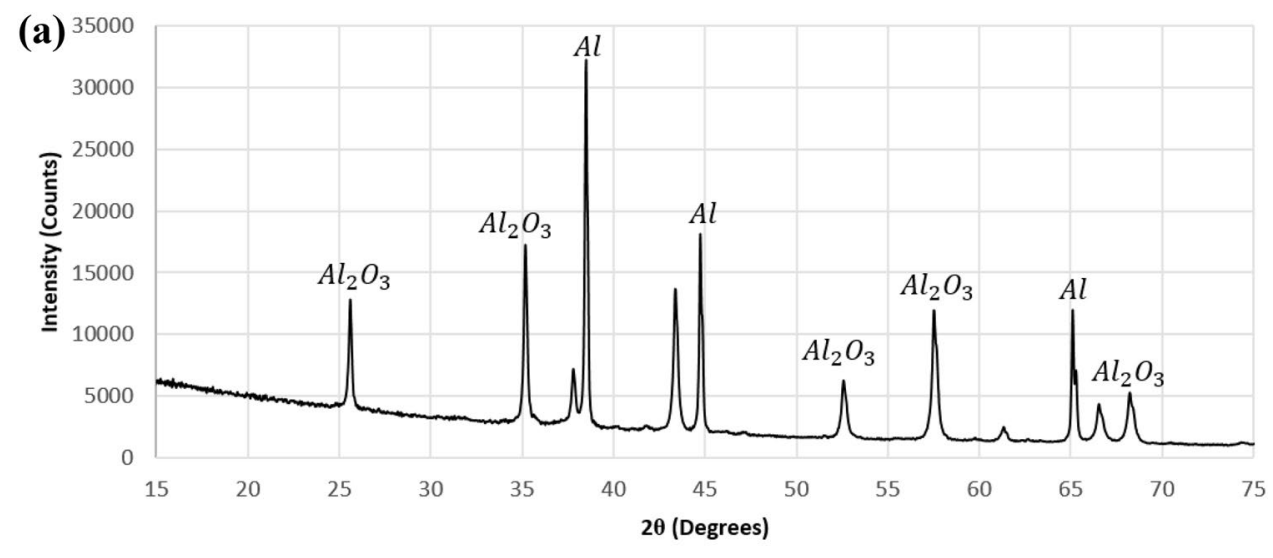

(b)

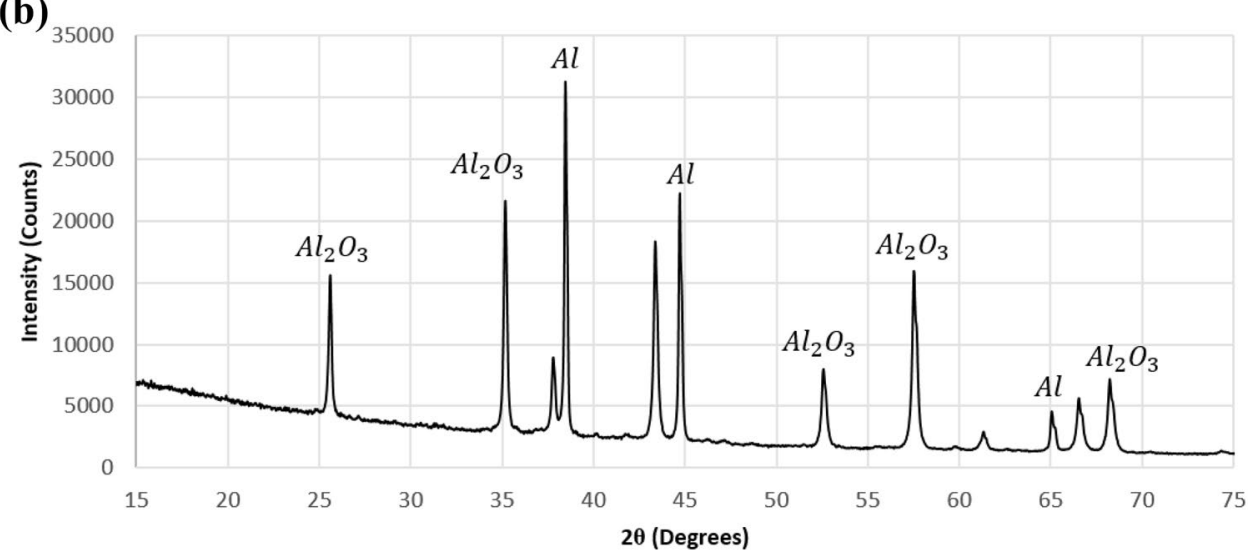

Table 1 Theoretical ceramic MGA physical properties at 50 vol\% Al

\begin{tabular}{lllll}
\hline System & $\begin{array}{l}\text { Phase } \\
\text { change } \\
\left({ }^{\circ} \mathrm{C}\right)\end{array}$ & $\begin{array}{l}\text { Density }(\mathrm{kg} / \\
\left.\mathrm{m}^{3}\right)\end{array}$ & $\begin{array}{l}\text { Energy } \\
\text { density } \\
(\mathrm{MJ} / \mathrm{L})\end{array}$ & $\begin{array}{l}\text { Thermal } \\
\text { conductivity at } \\
25^{\circ} \mathrm{C}(\mathrm{W} / \mathrm{mK})\end{array}$ \\
\hline $\mathrm{AIN}-\mathrm{Al}$ & 660 & 2980 & 0.91 & $104-202$ \\
$\mathrm{Al}_{2} \mathrm{O}_{3}-\mathrm{Al}$ & 660 & 3325 & 0.958 & $45-69$ \\
$\mathrm{MgO}-\mathrm{Al}$ & 660 & 3140 & 0.948 & $61-104$
\end{tabular}

still possesses many positive characteristics such as high thermal conductivity and energy density, and its use as a TES medium is still possible either within a protective environment or by refinements to the manufacturing process that eliminate open porosity.

The MgO-Al and the $\mathrm{Al}_{2} \mathrm{O}_{3}-\mathrm{Al}$ MGA systems showed great promise as oxygen-resistant MGA. These MGA showed no change after $72 \mathrm{hrs}$ at $700{ }^{\circ} \mathrm{C}$, as is evident
Fig. 8 AIN-Al (blue), MgO-Al (red) and $\mathrm{Al}_{2} \mathrm{O}_{3}-\mathrm{Al}$ (black) specific Heat proportional to Temperature

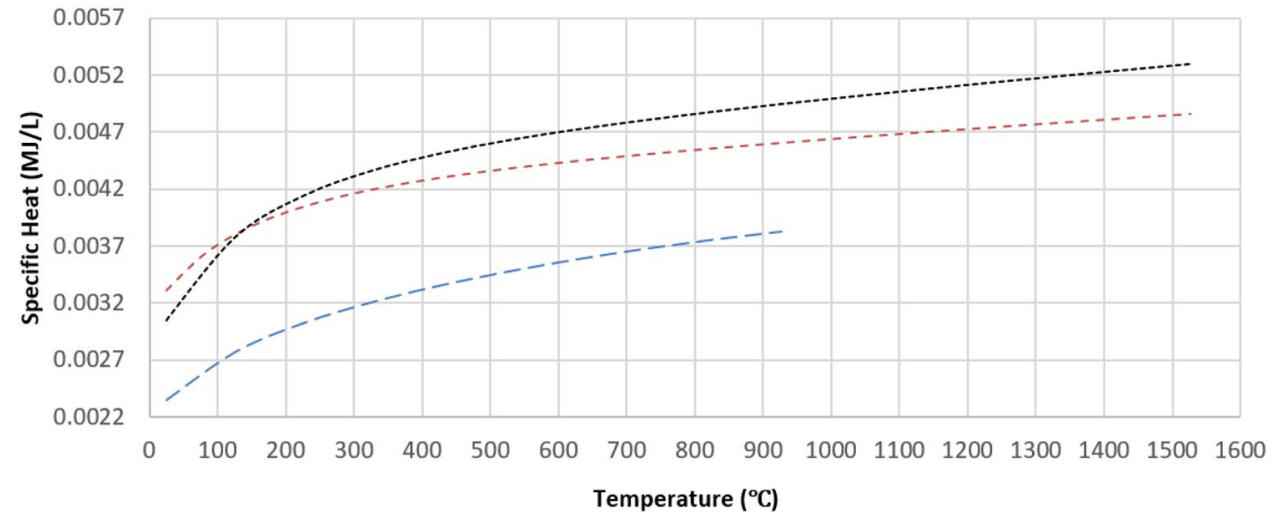

SN Applied Sciences 
Fig. 9 AlN-Al (blue), $\mathrm{MgO}-\mathrm{Al}$ (red) and $\mathrm{Al}_{2} \mathrm{O}_{3}-\mathrm{Al}$ (black) energy density curves relative to temperature

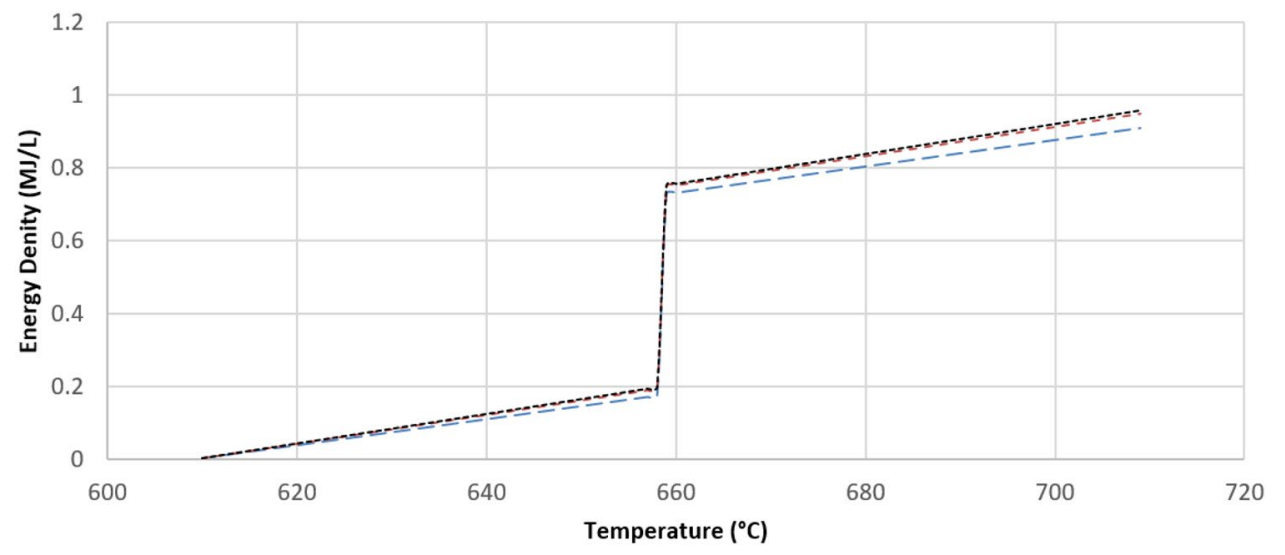

in Figs. 6 and 7. Those figures show near-identical X-ray diffraction patterns from the two MGA systems in the as-sintered and the oxidation-treated state. The microstructure of the MgO-Al MGA shown in Fig. 5 presents an ideal microstructure with a homogenous distribution of $\mathrm{Al}$ spheres embedded in an MgO matrix. Complete encapsulation of the Al particles is evident, with few micro-pores visible.

After oxidation, three of the MGA candidates underwent thermal testing. Figure 2 shows the endotherms of the AIN-Al, MgO-Al and the $\mathrm{Al}_{2} \mathrm{O}_{3}-\mathrm{Al}$ MGA, respectively. All three MGA candidates showed strong endotherms after sintering. As mentioned previously, the Al in the AIN MGA was susceptible to oxidation, and this is reflected in Fig. 2a), which shows the reduced endothermic response of the MGA after its oxidation treatment. The endothermic response from the other two MGA remained virtually unchanged between their as-sintered and oxidationtested states. This indicates that the oxidation process had no apparent detrimental effect on their energy absorption characteristics, suggesting that the goal of identifying oxidation-resistant MGA candidates has been achieved.

The hallmarks of MGA materials are that they are macroscopically solid over their operational range, deliver heat by conduction and possess high energy density due to an internal phase change. The theoretical properties of the new MGA systems shown in Table 1 are quite promising in this regard. Energy density approaching $1 \mathrm{MJ} / \mathrm{L}$, high thermal conductivity and a phase change centred around $660{ }^{\circ} \mathrm{C}$ make the materials useful for CSP applications where high-temperature steam is used in a Rankine cycle steam turbine and generator for electricity generation. Of these properties, the mass density and energy density are affected by processing factors which lead to porosity. Although it is possible to attain close to theoretical density in small laboratory samples, it is expected that the physically realised values may be as much as $10 \%$ below the values listed in Table 1 for storage modules rapidly manufactured at large scale as required for commercial exploitation.

The thermal conductivity, also given in Table 1, is the least certain of these predicted properties. Transport properties such as thermal conductivity are not only a function of the properties of the respective constituents and their relative fractions, but also microstructural features such as the shape and orientation of the included phase change particles (Al in this case) $[22,26]$. As the microstructure in Fig. 5 shows, the aim of roughly spherical particles well separated by the matrix material has been well approximated in these materials. Under these conditions, the uncertainty in the predicted thermal conductivities due to microstructural effects is in the range $10-20 \%$. A greater uncertainty enters due to the range of reported conductivity for the constituent materials, $\mathrm{Al}_{2} \mathrm{O}_{3}$ reportedly spanning $12-40 \mathrm{~W} / \mathrm{mK}$ and $\mathrm{MgO} 30-40 \mathrm{~W} / \mathrm{mK}$. AIN is more conductive than either of the other ceramics; however, it also has reported values over a wide range. Single crystals of AIN show conductivity as high as $285 \mathrm{~W} / \mathrm{mK}$, whereas polycrystalline ceramics are lower; values ranging from 60 to $177 \mathrm{~W} / \mathrm{mK}$ were used in calculating the values in Table 1.

There are many hundreds of potential MGA systems. From these, the features of those chosen for development are that they involve common and safe materials, have high energy density with a phase change at a useful temperature, possess high thermal conductivity and do not have very high material costs. In comparison with the graphite-based C-AI MGA $[13,17]$, the cost of the MgO and the $\mathrm{Al}_{2} \mathrm{O}_{3} \mathrm{MGA}$ is likely to be similar as the ceramic powders are relatively cheap ( $\$ 500-\$ 800$ per tonne). By comparison, AIN is more expensive and it is likely that the cost of AIN-AI MGA would be 2 to 4 times higher than the other two ceramic systems investigated here. Given the significant savings on infrastructure, maintenance and operating costs that a macroscopically thermal solid storage system may offer, there is likely to still be a role for AIN-Al in the future of CSP. 


\section{Conclusions}

This work has presented new, potentially oxygen-resistant MGA candidates for use as TES modules in CSP plants. Both the $\mathrm{MgO}$ - and the $\mathrm{Al}_{2} \mathrm{O}_{3}$-based MGA showed positive characteristics after oxidation, with minimal changes in their respective diffraction patterns and their DTA plots. Further work is currently being conducted on increasing the scale of these MGA systems, using higher firing temperatures, warm pressing the AIN-based systems and an extended oxidation test to understand the longevity of these MGA candidates. Along with understanding the oxidation kinetics of ceramic MGA systems, extended thermal cycling is currently underway on 19-mm and 32-mm ceramic MGA modules to understand their cyclic lifetime; it is believed that $>1000$ thermal cycles is required before a conclusion on ceramic MGA can be decided upon.

Acknowledgements The first author acknowledges receipt of a Research Training Program (RTP) Scholarship, provided by the University of Newcastle, and the Australian Government Department of Education and Training. At the University of Newcastle, the authors would like to thank the staff of the Mechanical Engineering Mechanical and Electrical Workshops for support with thermal testing and access to laboratory equipment. The authors would also like to thank Mrs. Jennifer Zobec and other staff of the Electron Microscope and $X$-ray Unit for access to X-ray diffraction equipment.

\section{Compliance with ethical standards}

Conflict of interest The authors declare that they have no competing interests.

\section{References}

1. Alva $G$, Lingkun $L$, Huang $X$, Fang $G$ (2017) Thermal energy storage materials and systems for solar energy applications. Renew Sustain Energy Rev 68:693-706

2. Alva G, Lin Y, Fang G (2018) An overview of thermal energy storage systems. Energy 144:341-378

3. Cunha JPD, Eames P (2016) Thermal energy storage for low and medium temperature applications using phase change materials - a review. Appl Energy 177(227-238):2016

4. Kearney D, Kelly B, Herrmann U, Cable R, Pacheco J, Mahoney R, Price H, Blake D, Nava P, Potrovitza N (2004) Engineering aspects of molten salt heat transfer fluid in a trough solar field. Energy 29(5-6):861-870

5. Khan Z, Khan Z, Ghafoor A (2016) A review of performance enhancement of PCM based latent heat storage system within the context of materials, thermal stability and compatibility. Energy Convers Manag 115:132-158

6. Xu B, Li P, Chan C (2015) Application of phase change materials for thermal energy storage in concentrated solar thermal power plants: a review to recent developments. Appl Energy 160:285-307
7. Reddy K, Mudgal V, Mallick T (2018) Review of latent heat thermal energy storage for improved material stability and effective load management. J Energy Storage 15:205-227

8. Narasimhan L (2019) Assessment of latent heat thermal storage systems operating with multiple phase change materials. J Energy Storage 23:442-455

9. Tao Y, He Y-L (2018) A review of phase change material and performance enhancement method for latent heat storage system. Renew Sustain Energy Rev 93:245-259

10. Gonzalez-Roubaud E, Perez-Osorio D, Prieto C (2017) Review of commercial thermal energy storage in concentrated solar power plants: steam vs. molten salts. Renew Sustain Energy Rev 80:133-148

11. Mohan G, Venkataraman MB, Coventry J (2019) Sensible energy storage options for concentrating solar power plants operating above $600^{\circ} \mathrm{C}$. Renew Sustain Energy Rev 107:319-337

12. Sugo H, Cuskelly D, Kisi E (2013) Miscibility gap alloys with inverse microstructure and high thermal conductivity for high energy density thermal storage applications. Appl Therm Eng 51(1-2):1345-1350

13. Reed S, Sugo H, Kisi E (2017) New highly thermally conductive thermal storage media. In: Sayigh A, Perth (eds) Transition towards $100 \%$ renewable energy. Springer, Cham

14. Rawson A, Sugo H, Kisi E, Fiedler T (2014) Thermal capacitors made from miscibility gap alloys (MGA). WIT Transact Ecol Eviron 186:479-486

15. Copus M, Fraser B, Reece R, Hands S, Cuskelly D, Sugo H, Reed S, Bradley J, Post A, Kisi E (2018) On-sun testing of miscibility gap alloy thermal storage. Sol Energy 177:657-664

16. Reed S, Sugo H, Kisi E (2018) High temperature thermal storage materials with high energy density and conductivity. Sol Energy 163:307-314

17. Reed S, Sugo H, Kisi E, Richardson P (2019) Extended thermal cycling of miscibility gap alloy high temperature thermal storage materials. Sol Energy 185:333-340

18. Copus M, Fraser B, Cuskelly D, Reed S, Post A, Kisi E (2019) Unifying capture, storage and discharge of thermal energy using miscibility gap alloys. AIP Conf Proc 2126(1):200012

19. Howard SM (1971) Ellingham diagrams. School of Mines and Technology, SD, Rapid City

20. Elorz JAP-S, Gonzalez DF, Verdeja LF (2019) Metals in structure materials. Springer, Cham, pp 19-76

21. Anghel C (2004) Studies of transport in oxides on Zr-based materials, Licentiate thesis, Royal Institute of Technology KTH, Stockholm, Sweden

22. Rawson A, Kisi E, Sugo H, Fiedler T (2014) Effective conductivity of Cu-Fe and Sn-Al miscibility gap alloys. Int J Heat Mass Transf 77:395-405

23. Touloukian YS (1970a) Thermophysical properties specific heat: metallic elements and alloys. Springer, NY

24. Touloukian YS (1970b) Thermophysical properties specific heat: nonmetallic solids. Springer, NY

25. Sugo H, Kisi E, Bradley J, Fiedler T, Luzin V (2017) In situ neutron diffraction studies of operating MGA thermal storage materials. Renew Energy Environ Sustain 2(34):5

26. Rawson A, Kisi E, Wensrich C (2015) Microstructural efficiency: structured morphologies. Int J Heat Mass Transf 81:820-828

Publisher's Note Springer Nature remains neutral with regard to jurisdictional claims in published maps and institutional affiliations. 